Physics Vol. 2, No. 5, pp. 205-207. 1966. Physics Publishing Co. Printed in Great Britain.

\title{
SOME REMARKS ON "IS THERE A FUNDAMENTAL DIFFERENCE BETWEEN A LIQUID AND A GAS?"
}

\author{
J. R. REITZ \\ Scientific Laboratory, Ford Motor Company, Dearborn, Michigan \\ (Received. 19 November 1965)
}

\begin{abstract}
The van der Waals equation is used to study the properties of a liquid-gas system in the region just above the critical point. The P-T curve of maximum isothermal compressibility, which persists to 1.068 times the critical temperature, is found to be a natural extension of the liquid-vapor equilibrium curve. Since the van der Waals equation is analytic, the present results do not support the view, recently advanced by Douglass, that there might be a fundamental difference between a liquid and a gas.
\end{abstract}

IN a recent paper Douglass [1] has raised some thought-provoking ideas concerning possible fundamental differences between a liquid and a gas in the region above the critical point where conventionally one regards the liquid-gas system as a single phase. He presents some excellent data on xenon by Habgood and Schneider [2] to show that the liquid-vapor equilibrium curve can be extended into the region in question, and that along this curve the isothermal compressibility of xenon has sharp maxima. If the extended line does, indeed, mark a transition between two phases, the transition would have to be of higher order since no latent heat or volume change is observed in this region of the liquid-gas system. The existence of a transition would, however, imply a different symmetry for the liquid and gas phases.

The purpose of the present note is to show that there are model systems for a liquid-gas which do not exhibit phase transitions in the region above the critical point, but which do show all of the features of the Habgood-Schneider data. From the excellent agreement of the data with the predictions of the model system one is forced to conclude that there is no evidence to support the idea of a fundamental difference between liquid and gas at temperatures above the critical temperature.

The model to be studied is the well-known van der Waals equation of state, which in reduced units may be written

$$
\left[P+3 / v^{2}\right][v-1 / 3]=(8 / 3) T .
$$

Here $P$ is the pressure in units of the critical pressure $P_{c}, T$ is the temperature in units of the critical temperature $T_{c}$, and $v$ is the volume in units of $V_{c}$. The van der Waals equation is analytic for $T \geqslant 1$; hence the isotherms in this region are characteristic of a single phase. 
However, for temperatures not too much greater than unity, these isotherms show maxima in the isothermal compressibility, $K_{T}=-\left(1 / P_{c} v\right)(d v / d P)_{T}$. It is easily verified that this effect persists to $T=3^{7} / 2^{11}$, or to 1.068 times the critical temperature. The maximum compressibility for a given isotherm is given by the equation,

$$
\left(d^{2} P / d v^{2}\right)_{T}=0 .
$$

For temperatures less than $T_{c}$, the van der Waals equation is usually altered by means of the Maxwell construction to replace the "unstable part" of the isotherm with a two-phase line. The $P-T$ relationship for the liquid-vapor equilibrium depends in detail upon the Maxwell construction, but for temperatures not too far below $T_{c}$ it may be approximated by the maximum slope in the "unstable part" of the analytic van der Waals equation. The liquid-vapor equilibrium curve is then specified by equation (2). Thus it is no accident that the curve of maximum compressibility above the critical point is an extension of the liquid-vapor curve.*

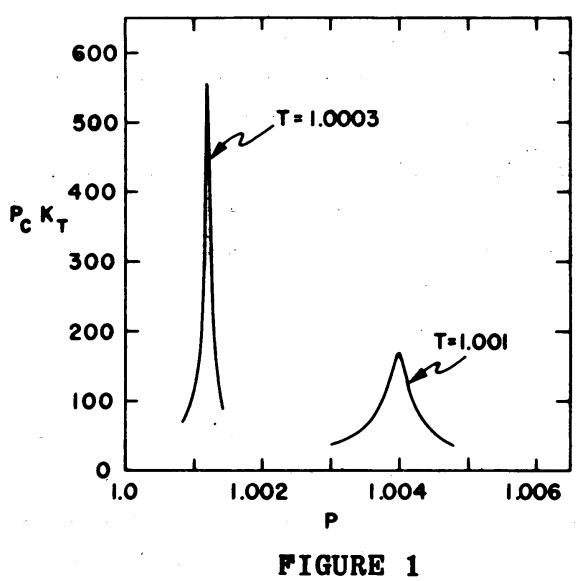

Plot of isothermal compressibility $K_{T}$ versus pressure for a van der Waals gas for two temperatures slightly above the critical temperature.

We have calculated the isothermal compressibility of a van der Waals gas in the region above the critical point directly from equation (1), and the results are shown in Fig. 1. The calculations were made for two temperatures in the range covered by the data of reference [1]. The similarity between the van der Waals results (Fig. 1) and the xenon data (Fig. 2 of Douglass' paper) is striking.

The van der Waals gas shows maxima in the compressibility which decrease as one moves away from the critical point, and disappear at a temperature about 7 percent greater than $T_{c}$. The maxima are all finite except for the $T_{c}$ isotherm. There are no indications in available data, e.g. [2], to support singularities in the compressibility, in fact, the data on xenon is in remarkable agreement with the van der Waals equation. The $P-T$ curve of maxima $K_{T}$ is a natural

* There are two inflection points on an isotherm for each $T<1.068$. It is the maximum compressibility point, or lower $v$ point, which falls on a $P-T$ curve extending from the 1 iquidvapor curve. 
extension of the liquid-vapor equilibrium curve. Thus we find no experimental support for the idea that liquid and gas represent different phases above the critical temperature.

\section{References}

1. D. H. DOUGLASS Jr., Physics 1, 329 (1965).

2. H.W. HABGOOD and W.G. SCHNEIDER, Can. J. Chem. 32, 98 (1954). 